\title{
The genome of the pear (Pyrus bretschneideri Rehd.)
}

Jun Wu, ${ }^{1,11}$ Zhiwen Wang, ${ }^{2,11}$ Zebin Shi, ${ }^{3,11}$ Shu Zhang, ${ }^{2,11}$ Ray Ming, ${ }^{4,11}$ Shilin Zhu, ${ }^{2,11}$ M. Awais Khan, ${ }^{5}$ Shutian Tao, ${ }^{1}$ Schuyler S. Korban, ${ }^{5}$ Hao Wang, ${ }^{6}$ Nancy J. Chen, ${ }^{7}$ Takeshi Nishio, ${ }^{8}$ Xun Xu, ${ }^{2}$ Lin Cong, ${ }^{2}$ Kaijie Qi, ${ }^{1}$ Xiaosan Huang, ${ }^{1}$ Yingtao Wang, ${ }^{1}$ Xiang Zhao, ${ }^{2}$ Juyou Wu, ${ }^{1}$ Cao Deng, ${ }^{2}$ Caiyun Gou, ${ }^{2}$ Weili Zhou, ${ }^{2}$ Hao Yin, ${ }^{1}$ Gaihua Qin, ${ }^{1}$ Yuhui Sha, ${ }^{2}$ Ye Tao, ${ }^{2}$ Hui Chen,, ${ }^{1}$ Yanan Yang, ${ }^{1}$ Yue Song, ${ }^{1}$ Dongliang Zhan, ${ }^{2}$ Juan Wang, ${ }^{2}$ Leiting Li, ${ }^{1,4}$ Meisong Dai, ${ }^{3}$ Chao Gu, ${ }^{1}$ Yuezhi Wang, ${ }^{3}$ Daihu Shi, ${ }^{2}$ Xiaowei Wang, ${ }^{2}$ Huping Zhang, ${ }^{1}$ Liang Zeng, ${ }^{2}$ Danman Zheng, ${ }^{5}$ Chunlei Wang, ${ }^{8}$ Maoshan Chen, ${ }^{2}$ Guangbiao Wang, ${ }^{2}$ Lin Xie, ${ }^{2}$ Valpuri Sovero, ${ }^{9}$ Shoufeng Sha, ${ }^{1}$ Wenjiang Huang, ${ }^{1}$ Shujun Zhang, ${ }^{3}$ Mingyue Zhang, ${ }^{1}$ Jiangmei Sun, ${ }^{1}$ Linlin Xu, ${ }^{1}$ Yuan Li, ${ }^{1}$ Xing Liu, ${ }^{1}$ Qingsong Li, ${ }^{1}$ Jiahui Shen, ${ }^{1}$ Junyi Wang, ${ }^{2}$ Robert E. Paull, ${ }^{7}$ Jeffrey L. Bennetzen, ${ }^{6}$ Jun Wang, 2,10,12 and Shaoling Zhang ${ }^{1,12}$

${ }^{1}$ Centre of Pear Engineering Technology Research, State Key Laboratory of Crop Genetics and Germplasm Enhancement, Nanjing Agricultural University, Nanjing 210095, China; ${ }^{2}$ BGI-Shenzhen, Shenzhen 518083, China; ${ }^{3}$ Institute of Horticulture, Zhejiang Academy of Agricultural Sciences, Hangzhou 310021, China; ${ }^{4}$ Department of Plant Biology, ${ }^{5}$ Department of Natural Resources and Environmental Sciences, University of Illinois, Urbana, Illinois 61801, USA; ${ }^{6}$ Department of Genetics, University of Georgia, Athens, Georgia 30602, USA; ${ }^{7}$ Department of Tropical Plant and Soil Sciences, University of Hawaii, Honolulu, Hawaii 96822, USA; ${ }^{8}$ Graduate School of Agricultural Science, Tohoku University, Aoba-ku, Sendai 981-8555, Japan; ${ }^{9}$ Department of Crop Sciences, University of Illinois, Urbana, Illinois 61801, USA; ${ }^{10}$ Department of Biology, University of Copenhagen, Copenhagen 2200, Denmark

The draft genome of the pear (Pyrus bretschneideri) using a combination of BAC-by-BAC and next-generation sequencing is reported. A $512.0-\mathrm{Mb}$ sequence corresponding to $97.1 \%$ of the estimated genome size of this highly heterozygous species is assembled with $194 \times$ coverage. High-density genetic maps comprising 2005 SNP markers anchored $75.5 \%$ of the sequence to all 17 chromosomes. The pear genome encodes 42,812 protein-coding genes, and of these, $\sim 28.5 \%$ encode multiple isoforms. Repetitive sequences of $271.9 \mathrm{Mb}$ in length, accounting for $53.1 \%$ of the pear genome, are identified. Simulation of eudicots to the ancestor of Rosaceae has reconstructed nine ancestral chromosomes. Pear and apple diverged from each other 5.4-21.5 million years ago, and a recent whole-genome duplication (WGD) event must have occurred 30-45 MYA prior to their divergence, but following divergence from strawberry. When compared with the apple genome sequence, size differences between the apple and pear genomes are confirmed mainly due to the presence of repetitive sequences predominantly contributed by transposable elements (TEs), while genic regions are similar in both species. Genes critical for self-incompatibility, lignified stone cells (a unique feature of pear fruit), sorbitol metabolism, and volatile compounds of fruit have also been identified. Multiple candidate SFB genes appear as tandem repeats in the S-locus region of pear; while lignin synthesis-related gene family expansion and highly expressed gene families of $H C T, C 3^{\prime} H$, and $C C O M T$ contribute to high accumulation of both G-lignin and S-lignin. Moreover, alpha-linolenic acid metabolism is a key pathway for aroma in pear fruit.

[Supplemental material is available for this article.]

Pear, the third most important temperate fruit species after grape and apple, belongs to the subfamily Pomoideae in the family Rosaceae. The majority of cultivated pears are functional diploids $(2 \mathrm{n}=34)$. As a popular fruit in the world market, pear has widespread cultivation on six continents, with major production in China, the United States, Italy, Argentina, and Spain (Supplemental Fig. 1).

\footnotetext{
${ }^{11}$ These authors contributed equally to this work.

${ }^{12}$ Corresponding authors

E-mail slzhang@njau.edu.cn

E-mail wangj@genomics.org.cn

Article published online before print. Article, supplemental material, and publication date are at http://www.genome.org/cgi/doi/10.1101/gr.144311.112. Freely available online through the Genome Research Open Access option.
}

Pears are among the oldest of the world's fruit crops, with $>3000 \mathrm{yr}$ of cultivation history (Lombard and Westwood 1987), likely originating during the Tertiary period (65-55 million years ago [MYA]) in the mountainous regions of southwestern China and, from there, spreading on to both the East and West (Rubtsov 1944; Zeven and Zhukovsky 1975). Central Asia and eastern China are identified as two subcenters of genetic diversity for pear (Vavilov 1951). The Pyrus genus is genetically diverse with thousands of cultivars, but it can be divided into two major groups, Occidental pears (European pears) and Oriental pears (Asiatic pears). At least 22 primary species are well-recognized in Pyrus; however, only a few species, including Pyrus bretschneideri, Pyrus pyrifolia, Pyrus ussuriensis, Pyrus sinkiangensis, and Pyrus communis, have been utilized for fruit production. 
Herein, we report on a high-quality draft genome sequence of the diploid P. bretschneideri Rehd. cv. 'Dangshansuli' (also known as 'Suli'), the most important commercial Asiatic pear cultivar grown in the world ( $>4$ million tons per year), having $>500 \mathrm{yr}$ of cultivated history in China. Pear is highly heterozygous due to selfincompatibility and interspecies compatibility. The genome is known to have an abundance of repetitive DNA sequences. In this study, a novel combination of BAC-by-BAC (bacterial artificial chromosome) strategy, with Illumina sequencing technology, is used for the first time for de novo assembly of a highly heterozygous genome of this size with highly repetitive DNA sequences. This has demonstrated that a complex plant genome sequence can be assembled and characterized using these technologies without the availability of a physical reference. Additionally, we also report on primary factors contributing to genome size differences between pear and apple, both belonging to the subfamily Pomoideae; chromosomal evolution of Rosaceae; and genes controlling valuable traits of pear, including self-incompatibility, lignified stone cells in flesh of fruit (unique to pear), sugar, and aroma.

\section{Results and Discussion}

\section{Sequencing a highly heterozygous genome}

The pear cultivar 'Suli' was first sequenced using a whole-genome shotgun (WGS) approach, but the quality of the assembled genome was poor. Analysis of 17-mer sequences revealed high levels of heterozygosity in the genome and a $1 \%-2 \%$ sequence divergence between alleles (Supplemental Fig. 2). To overcome this, a BAC-by-BAC strategy was used instead to sequence and assemble the pear genome. A total of 38,304 BACs was selected for sequencing, representing $7.6 \times$ genome equivalents. Two paired-end libraries with insert sizes of $250 \mathrm{bp}$ and $500 \mathrm{bp}$, respectively, were constructed for each BAC, and sequenced at a combined $86 \times$ coverage using Illumina HiSeq 2000 (Supplemental Table 1). Each BAC was assembled individually prior to attempting wholegenome assembly. In addition, WGS mate-pair libraries of $2 \mathrm{~kb}$, $5 \mathrm{~kb}, 10 \mathrm{~kb}, 20 \mathrm{~kb}$, and $40 \mathrm{~kb}$ were constructed and sequenced at $24 \times$ coverage to build super-scaffolds; moreover, paired-end libraries of 180,500 , and 800 bp were constructed and sequenced at $83 \times$ coverage to fill in gaps (Supplemental Table 2). All BAC sequences were pooled for overlap-layout-consensus (OLC) assembly, identical sequences were merged, and redundant bases were filtered out from overlapping lengths. The resulting contigs were assembled into scaffolds by WGS pairedend reads of large-insert libraries (2-40 $\mathrm{kb}$ ), and gaps were filled with WGS paired-end reads of small-insert libraries (180-800 bp).

The quality of the assembly was assessed by aligning scaffolds to five fully assembled BAC sequences. The coverage ratios of BAC1, BAC2, BAC4, and BAC5 were $>98 \%$ with good synteny of scaffolds (Supplemental Table 3; Supplemental Fig. $3)$, while the coverage ratio of BAC3 was $90 \%$ as it had a $12 \mathrm{~K}$ fragment that did not align to scaffold 227.0. This was attributed to differences between the two haplotypes. One haplotype was assembled in the final scaffold, but BAC3 belonged to an unassembled haplotype, even though both have been assembled in the BAC-to-BAC assembly step (Supplemental Fig. 4).

The assembled pear genome consists of 2103 scaffolds with $\mathrm{N} 50$ at $540.8 \mathrm{~kb}$, totaling $512.0 \mathrm{Mb}$ with $194 \times$ coverage, close to the estimated size of $527 \mathrm{Mb}$ (Table 1). Among 2005 SNP markers in the genetic map, $100 \%$ of the SNPs are anchored to 796 scaffolds, $386.7 \mathrm{Mb}$, representing $\sim 75.5 \%$ of the assembled genome (Supplemental Fig. 5).

\section{Heterozygozity features of the pear genome}

A total of 3,402,159 reliable SNPs were identified in 'Suli.' By use of the same filtering standard, 333,443,735 reliable genome bases were identified; thus, the frequency of SNPs in this genome was $\sim 1.02 \%$. Heterozygosity of pear was higher than that of other plants, such as papaya (0.06\%) (Ming et al. 2008), pigeonpea $(0.067 \%)$ (Varshney et al. 2012), black cottonwood (0.26\%) (Tuskan et al. 2006), and date palm (0.46\%) (Al-Dous et al. 2011), but heterozygosity was lower than that of grape (7\%) (Jaillon et al. 2007). The distribution profile of SNPs showed that $87.1 \%$ of SNPs were within $50 \mathrm{bp}$ of each other, and nearly $50 \%$ were within $<10$ bp from an adjacent SNP (Supplemental Fig. 6). In contrast to frequency of SNPs within the whole genome, genes had lower frequencies of SNPs, $0.84 \%$, along with $0.70 \%$ for coding sequence (CDS), $0.95 \%$ for introns, and $0.90 \%$ for UTRs. It was assumed that the frequency of SNPs in CDS was attributed to its conserved protein-coding function. A total of 26,249 genes had SNPs (Supplemental Fig. 7). Of those, 13,794 genes had SNPs of $<1 \%$, and 4346 genes had SNPs of $>2 \%$. These genes were enriched in major functional categories, including protein kinase, disease resistance protein, cell division protein, ion transfer, and transcription factor. Genes with significantly high frequencies $(>20 \%)$ of SNPs belonged to those with basic functions, including membrane, cell wall, cell division, and methylation, among others (Supplemental Fig. 8). Due to presence of SNPs, 1300 genes changed from coding for amino acids to stop codons (nonsense mutations), and 500 genes changed from stop codons to other amino acids; these genes were enriched for biological function, including cell division, protein kinase, and WD40 protein.

\section{Influence of repetitive sequences on genome size variation}

A combination of structure-based analysis and homology-based comparisons identified $271.9 \mathrm{Mb}$ repetitive sequences, accounting for $53.1 \%$ of the current assembly of the pear genome (Supple-

Table 1. Summary of genome assembly features and annotation of the pear (Pyrus bretschneideri Rehd.) genome sequence

\begin{tabular}{llrcccc}
\hline $\begin{array}{l}\text { Unit of } \\
\text { assembly }\end{array}$ & $\begin{array}{l}\text { Proportion/ } \\
\text { unit type }\end{array}$ & \multicolumn{1}{c}{ No. } & \multicolumn{1}{c}{ Size } & \% assembly & $\begin{array}{r}\text { N50 } \\
(\mathbf{k b})\end{array}$ & $\begin{array}{c}\text { Longest } \\
\mathbf{( M b )}\end{array}$ \\
\hline Contigs & All & 25,312 & $501.3 \mathrm{Mb}$ & 97.9 & 35.7 & 0.3 \\
Scaffolds & All & 2,103 & $512.0 \mathrm{Mb}$ & 100 & 540.8 & 4.1 \\
& Anchored & 796 & $386.7 \mathrm{Mb}$ & 75.5 & 698.0 & 4.1 \\
Genes & Total & 42,812 & $118.8 \mathrm{Mb}$ & 23.2 & & \\
& Exon & 202,169 & $50.2 \mathrm{Mb}$ & 9.8 & & \\
& Intron & 159,357 & $61.2 \mathrm{Mb}$ & 11.9 & & \\
ncRNA & miRNA & 297 & $37,168 \mathrm{bp}$ & 0.01 & & \\
& tRNA & 1,148 & $86,791 \mathrm{bp}$ & 0.02 & & \\
& rRNA & 697 & $228,388 \mathrm{bp}$ & 0.04 & & \\
& SnRNA & 395 & $45,301 \mathrm{bp}$ & 0.01 & & \\
Repetitive & & & $271.9 \mathrm{Mb}$ & 53.1 & & \\
sequences & & & & & &
\end{tabular}


mental Table 4). The most abundant transposon families were sypsy and copia, contributing for $25.5 \%$ and $16.9 \%$ of the genome, respectively (Supplemental Table 5). Long terminal repeat (LTR) retrotransposons exhibited family-specific, nonuniform distributions along chromosomes. Copia-like elements were spread along the whole chromosome, including gene-rich euchromatic regions, whereas gypsy-like elements were overrepresented in gene-poor heterochromatic regions (Fig. 1, Supplemental Fig. 9). The most abundant DNA transposable elements (TEs) were PIF/Harbinger and hAT-Ac elements, representing $2.7 \%$ and $2.1 \%$ of nuclear DNA, respectively (Supplemental Table 5). Although widely dispersed throughout the genome, transposon-related sequences were most abundant in centromeric regions (Fig. 1; Supplemental Fig. 9).

Structural searches identified 645 reliable intact TEs and 19 Solo-LTRs in pear (Supplemental Table 6). These intact elements masked $34.0 \%$ of the assembly, accounting for $\sim 70.0 \%$ of the total repeats. Of 299 intact LTR retrotransposons, 144 belonged to the copia superfamily and 31 to the gypsy superfamily. Low numbers of intact gypsy elements did not suggest that gypsy elements were relatively rare as large numbers of gypsy RT domains were detected. By searching flanking sequences of DDE and RT domains, 10 intact hAT, eight PIF/Harbinger, two CACTA, 38 LINEs, and 288 MITE elements (yielding 221 exemplars) were found (Supplemental Table 7). A partial reason for detecting relatively few intact TEs (357, excluding MITEs) was due to incomplete sequencing of individual BACs, thus leaving gaps in the assembly (especially in terminal repeats). This suggested that an element could not be deemed intact by any of the structure-based search algorithms used. However, these methods yielded $\sim 3000$ intact TEs in a $\sim 500-\mathrm{Mb}$ WGS assembly of the genome of the grass species Setaria italica (Bennetzen et al. 2012). Thus, it was likely that the pear genome yielded few intact elements in this current analysis either due to abundance of elements that were structurally rearranged or due to presence of more than a single copy of elements of the same families on any given BAC. This could be attributed to yield of high copy numbers of many families and/or insertion preferences during clustering, as noted for Helitrons in maize (Yang and Bennetzen 2009).

Of a total of $603.9 \mathrm{Mb}$ assembled data of the apple genome, an estimated $362.3 \mathrm{Mb}$ repetitive sequences has been reported (Velasco et al. 2010). However, the nonrepeat region of apple and pear is of almost equal size (241.6 Mb for apple and 240.2 Mb for pear). Thus, the difference in repetitive sequences of assembled sequences of apple and pear is $90 \mathrm{Mb}$, mainly consisting of two forms of TEs, gypsy and LINE (Fig. 2). Additionally, a large portion of unassembled sequences in apple has been deemed as repetitive sequences (Velasco et al. 2010). Assembly of highly repetitive sequences is a major limitation for de novo sequencing of a heterozygous genome, such as pear, using WGS and next-generation sequencing technologies (Birney 2010). This is particularly true for TE families that have undergone recent amplifications. The BAC-byBAC approach used in this study has ensured a relatively accurate assembly of TEs in the pear genome as TEs in different BACs would have rare effects during assembly of these BACs, although assembly of fully intact elements will be rare either when TEs contain terminal repeats (e.g., LTR retrotransposons) or when more than a single copy of the same TE is found in a specific BAC. Based on these findings, observed genome size differences between apple and pear are mainly due to repetitive sequences predominantly contributed by TEs, while genic regions are similar in both species.

LTRs with complete structures in pear are predicted to estimate insertion time via distances between $5^{\prime}$ and $3^{\prime}$ solo-LTRs.
These findings indicate that pear has a high LTR expansion rate, wherein a recent twofold increase in LTR numbers must have occurred, compared with that of other sequenced plant species (The Arabidopsis Genome Initiative 2000; Ming et al. 2008; Shulaev et al. 2010; Velasco et al. 2010). This suggests that the pear genome is in continuous expansion (Supplemental Fig. 10). However, these results may also be influenced by the method of assembly.

\section{Gene annotation and transcriptome sequence analysis}

By combining ab initio gene prediction and protein alignment prediction, 42,767 protein-coding genes were annotated. Comparisons of transcriptome sequences to gene models using Illumina RNA-seq sequences provided empirical support for these predictions. This gene prediction approach proved highly effective, as $23,843(55.7 \%)$ hybrid gene models were supported by 25,365 (93.9\% of 27,008 transcripts with complete open reading frames [ORFs]) transcript-based sequences (Supplemental Fig. 11). After integrating and then adding novel transcriptome-based genes, a total of 58,596 transcripts constituted 42,812 gene loci, among which 12,217 (28.5\%) genes encoded multiple isoforms. Thus, gene prediction based on whole-genome assembly in pear was credible. On average, gene models consisted of transcript lengths of $2776 \mathrm{bp}$, coding lengths of $1172 \mathrm{bp}$, and means of 4.7 exons per gene, both similar to those observed in apple (Velasco et al. 2010) and Arabidopsis (The Arabidopsis Genome Initiative 2000 ). A total of $89.5 \%$ of gene models had matches in at least one of the public protein databases. These findings also confirmed completeness of the pear genome sequence coverage. In addition, 297 microRNAs (miRNAs), 1148 transfer RNAs (tRNAs), 697 ribosomal RNAs (rRNAs), and 395 small nuclear RNAs (snRNA) were identified in the pear genome (Table 1).

The number of genes found in pear is similar to that found in other sequenced plants of equivalent genome size but is much lower than that of the closely related apple genome (Velasco et al. 2010). The pear genome has been sequenced using a BAC-by-BAC approach, resolving problems of assembling a heterozygous genome. In contrast, the apple genome has been sequenced using a WGS approach, wherein some alleles may have been annotated as individual genes. This is demonstrated by alignment of a single unique chromosome region to two overlapping scaffolds in apple (Supplemental Fig. 12). The assembly of two scaffolds for a single genomic region resulted in overestimation of the assembled genome and gene numbers in apple. After filtering out of overlapping genes in apple chromosomes, the gene number in apple dropped from either 61,334 (based on NCBI) or 57,386 (based on the published report; Velasco et al. 2010) down to 45,293 . This indicates that the numbers of genes in apple and pear are almost equal.

The average gene density in pear is one gene per $12 \mathrm{~kb}$, with genes being more abundant in subtelomeric regions (Fig. 1; Supplemental Fig. 9), as previously observed in other sequenced plant genomes. Gene elements in pear, including lengths of miRNAs, distribution of CDS, and exons and introns, are normally distributed compared with those of five other plant species, including apple (Malus $\times$ domestica) (Velasco et al. 2010), strawberry (Fragaria vesca) (Shulaev et al. 2010), Arabidopsis (Arabidopsis thaliana) (The Arabidopsis Genome Initiative 2000), grape (Vitis vinifera) (Jaillon et al. 2007), and black cottonwood (Populus trichocarpa) (Tuskan et al. 2006) (Supplemental Fig. 13). Moreover, the pear genome maintains higher numbers of genes for transport and catalysis within the 'molecular function' gene ontology (GO) category; for cellular process, protein metabolism, and biological regulation

\section{Genome Research}



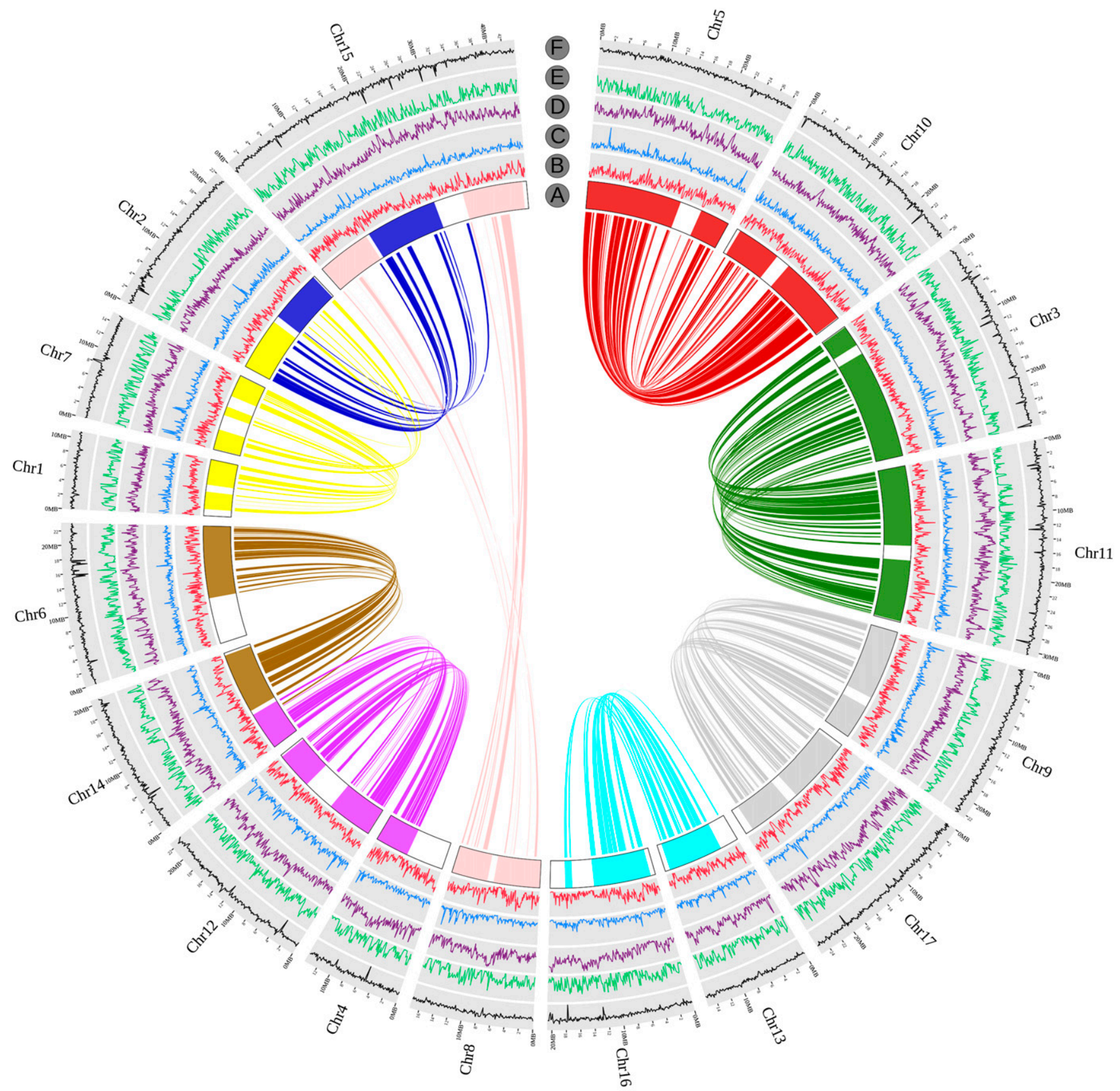

\section{A Chromosome karyotype} Rosaceae Ancestors

B Gene density(per 100kbp)

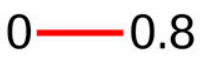

C DNA TE density(per 100kbp)

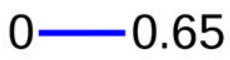

D RT TE density(per 100kbp)

$0-1$

E SNP density(per 100kbp)

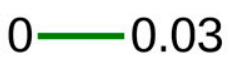

F GC content(per 100kbp)

$0.25-0.45$

Figure 1. Distribution of basic genomic elements of pear. $(A)$ Chromosome karyotype. Colored segments are in accordance with the Rosaceous ancestor. (B) Gene density. The rate of sites within gene region per $100 \mathrm{~kb}$ ranges from a minimum of 0 to a maximum of 0.8 , illustrated by red line. (C) DNA transposon element (TE) density. The rate of sites within the DNA TE region per $100 \mathrm{~kb}$ ranges from 0 to 0.65 , illustrated by blue line. (D) Retrotransposon element (RT TE) density. The rate of sites within the RT TE regions ranges from 0 to 1 , illustrated by purple. (E) SNP density. The rate of SNP per $100 \mathrm{~kb}$ ranges from 0 to 0.03 , illustrated by green. $(F) \mathrm{GC}$ content. The rate of GC content ranges from 0.25 to 0.45 , illustrated by black. Circos (Krzywinski et al. 2009) (http://circos.ca) was used for constructing this diagram. 


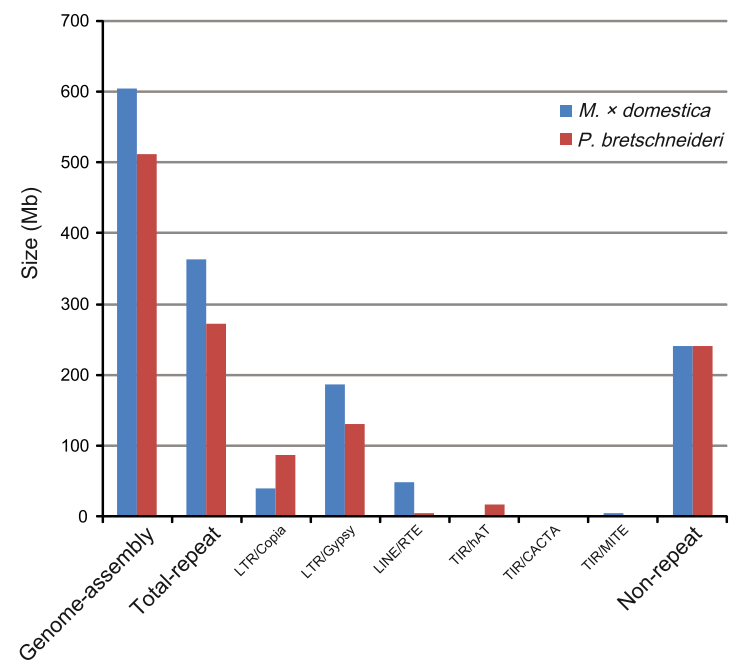

Figure 2. Comparisons between apple and pear for repetitive elements. The major repeats in apple and pear revealed that genome size differences of apple and pear were mainly attributed to repeat sequences.

within the 'biological processes' GO category; and for cell, intracellular, and membrane within the 'cellular component' GO category (Supplemental Fig. 14).

\section{Whole-genome duplication and divergence of Pyrus}

A total of 13,372 pairs of paralogous genes in pear are aligned in 870 blocks (seven or more gene pairs per block). A fourfold degenerate site transversion ( $4 \mathrm{dTv}$ ) of these blocks was calculated and was corrected by HKY. From the distribution of $4 \mathrm{dTV}$ (Figure 3), it can be concluded that there are two significant groups of blocks, suggestive of whole-genome duplication (WGD) events in pear, including a recent event with $4 \mathrm{dTv}$ of $\sim 0.08$ and an ancient event with $4 \mathrm{dTv}$ of $\sim 0.5$. Both WGD events are shared by apple and pear, but strawberry does not share the recent WGD event. Distribution of $4 \mathrm{dTv}$ values suggests that divergence between pear and apple must have occurred after the recent WGD event. To estimate the time of occurrence of these two duplication events, a total of 16,335 paralogous gene pairs within 5593 gene families with substitutions per synonymous site (Ks) values lower than two are selected. Based on Ks values in pear, the main peak ranges from $0.15-0.3$, while the secondary peak ranges from 1.5 to 1.8 , similar to that found in apple (Supplemental Fig. 15A,B). As in apple, the recent WGD in pear must have occurred at 30-45 MYA (Velasco et al. 2010), while the ancient WGD must have resulted from an acknowledged paleohexaploidization event that took place $\sim 140$ MYA (Fawcett et al. 2009). The divergence time of eight sequenced plant species (The Arabidopsis Genome Initiative 2000; Khurana and Gaikwad 2005; Tuskan et al. 2006; Jaillon et al. 2007; Ming et al. 2008; Shulaev et al. 2010; Velasco et al. 2010), including pear, apple, strawberry, papaya, grape, black cottonwood (poplar), Arabidopsis, and rice, has been estimated according to known ranges of divergence time and the phylogenetic tree (Supplemental Fig. 16A). It is estimated that pear and apple must have diverged from each other 5.4-21.5 MYA (Supplemental Fig. 16B).

\section{Evolution of chromosomes in Rosaceae}

Collinearity analysis between pear and two sequenced rosaceous species, apple and strawberry, has revealed that pear and apple share similar chromosome structures as well as organization
(Supplemental Fig. 17A,B). All 17 chromosomes of pear displayed good homology with corresponding chromosomes of apple (Supplemental Fig. 17A). Based on self-collinearity of pear (Fig. 1A; Supplemental Fig. 17B), it is easy to identify good syntenic chromosome pairs in pear, such as LG3 and LG11, LG5 and LG10, LG9 and LG17, and LG13 and LG16, as well as rearrangement of chromosomes as identified in the apple genome (Velasco et al. 2010). Most collinear regions between pear and strawberry reveal that one chromosome in strawberry corresponds to two chromosomes in pear (Supplemental Fig. 17C, 1,2). For example, LG1 in strawberry corresponds to Chr2 and Chr15 in pear, and similarly LG2 to Chr5 and Chr10, LG3 to Chr3 and Chr11, LG4 to Chr13 and Chr16, as well as LG5 to Chr6 and Chr14, respectively. It appears that LG2, LG3, LG5, and LG6 of strawberry are formed by fragmentation and recombination of ancestral chromosomes (Fig. 4). In this study, ancestral chromosomes of paleohexaploid eudicots have been reconstructed to the ancestor of Rosaceae by collinearity between strawberry and grape genomes (Supplemental Fig. 17D). Using relationships of strawberry and Rosaceae and those of strawberry and eudicots, a simulated process of eudicots to Rosaceae has been developed. Results have revealed that triplication of seven ancestral chromosomes of eudicots may have undergone additional rearrangements, yielding nine ancestral chromosomes of Rosaceae (Fig. 4).

\section{Disease resistance-related genes}

A total of 396 nucleotide-binding site (NBS)-containing $R$ genes were identified in pear (Supplemental Table 10). This was similar to that found in soybean (Glycine max; 392) and in poplar (402), and $\sim 39.9 \%$ and $74.0 \%$ of that found in apple (992) and in rice (Oryza sativa) (535), respectively, but higher than that detected in Eurosids II, including both cacao (Theobroma cacao) (253) and Arabidopsis (178). However, the observed twofold difference of numbers of

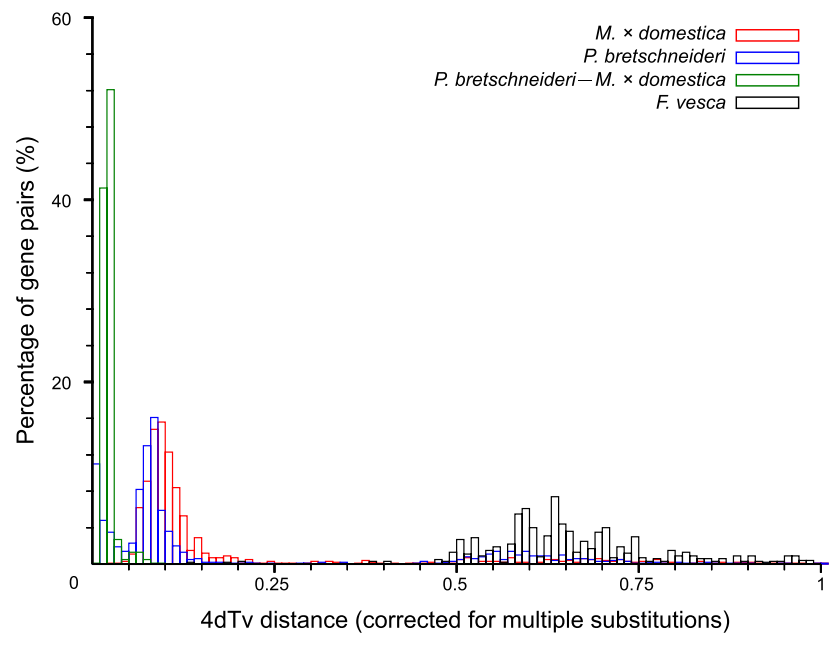

Figure 3. Distribution of fourfold degenerate site (4dTv) distances of duplicate gene pairs in pear, apple, and strawberry. A total of 1085 synteny blocks in pear (726 in apple and 262 in strawberry) are selected to calculate $4 \mathrm{dTv}$ values. The distribution of $4 \mathrm{dTV}$ values in pear (in blue) and those of apple (in red) are similar, while those of strawberry (in black) are different, with a single peak around 0.65 , suggesting that there is no recent whole-genome duplication (WGD) in strawberry. (Green groups) Synteny blocks (557) between pear and apple, revealing that these groups are closer to the $y$-axis, and suggesting a more recent divergence event must have occurred between pear and apple.

\section{Genome Research}



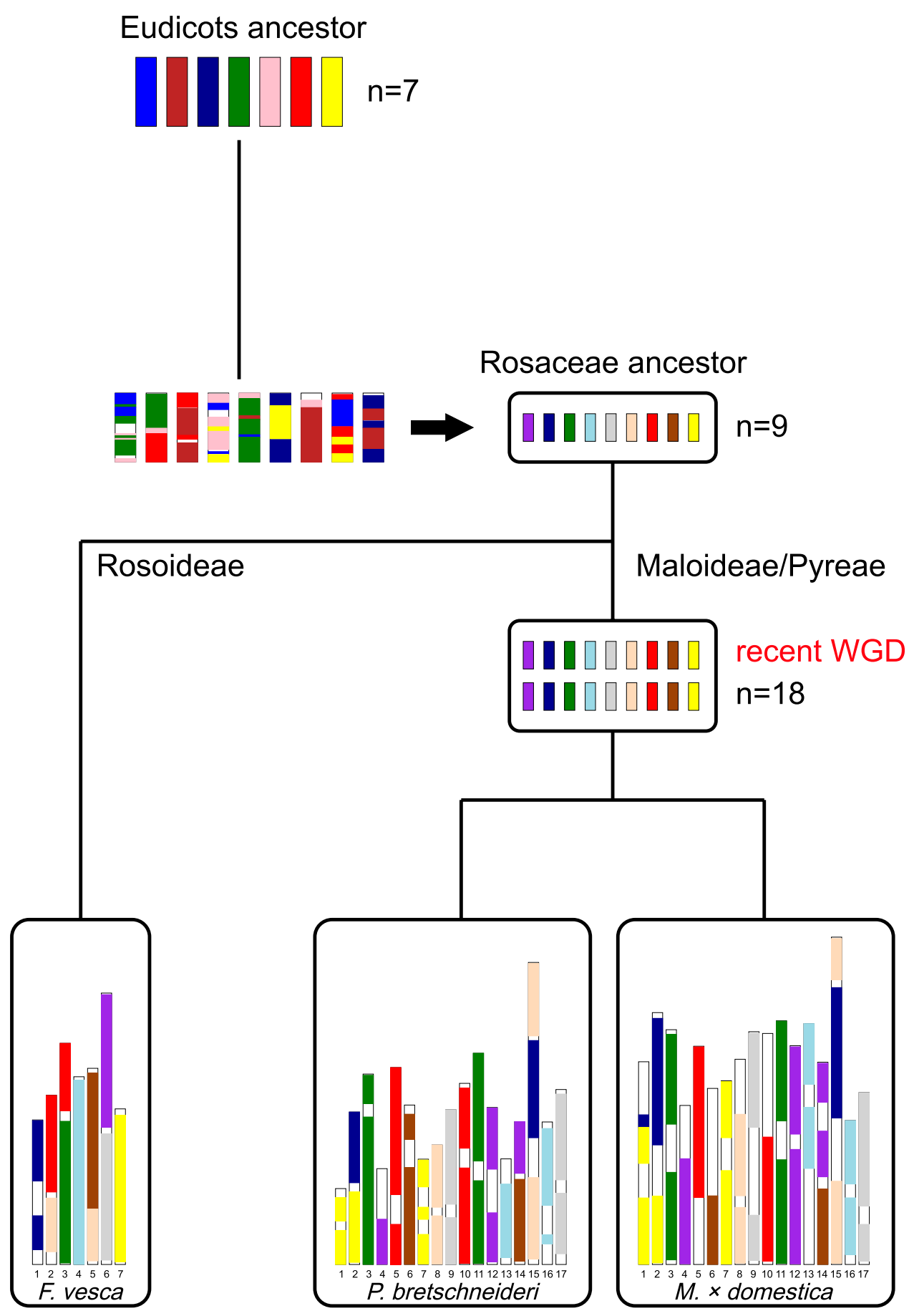

Figure 4. The evolutionary scenario of nine chromosomes of the Rosaceae ancestor. Pear and apple have the same chromosome karyotypes and same chromosomal evolution mode. The Pyreae tribe went through a recent WGD. The Amygdaleae tribe, such as strawberry in the Rosoideae subfamily, has no recent WGD but has chromosome fragmentation and recombination from nine to seven. It is estimated that the ancestor of Rosaceae had nine chromosomes. To demonstrate the evolutionary process from the eudicot ancestor to the Rosaceae ancestor, strawberry was compared with grape.

$R$ genes between pear and apple might have been overestimated. Furthermore, pear CC-NBS-LRR genes outnumbered TIR-NBSLRRs, which were similar to that observed in both grape and poplar, but in contrast to that found in apple, soybean, and Arabidopsis. In addition to NBS genes, the pear genome contained 403 LRR-kinase genes and 11 additional CC-LRR-Kinase genes, which is higher than that found in both apple (320) and poplar (269).
When the $R$ paralogous genes were mapped along pear pseudomolecules, they were found to be nonrandomly distributed across all 17 chromosomes (Supplemental Fig. 18). More than 30\% of $R$ genes were clustered in groups (Supplemental Fig. 19), and clusters were most abundant on chromosomes 2, 5, and 11 (Supplemental Fig. 20). Enrichment of $R$ genes in these corresponding genomic regions indicated that resistance gene evolution might 
involve tandem duplication and divergence of linked gene families, similar to those found in other known plant genomes.

\section{S-locus comparisons in gametophytic self-incompatible species}

Pear exhibits typical gametophytic self-incompatibility (GSI) controlled by an apparently single multi-allelic locus (the $S$-locus) (de Nettancourt 1997) containing at least two linked genes: one is a pistil $S$-determinant, known as $S$-RNase gene (Ushijima et al. 2003), and the other is a pollen $S$-determinant, proposed as an $S$-haplotype-specific F-box (SFB) protein and identified in Prunus species (Zhang et al. 2007; Wu et al. 2009). However, candidates for $S F B$ genes controlling pollen self-incompatibility in pear have remained unclear until now. Based on the assembled sequence of the pear genome, the $S$-locus is anchored close to the end region of 3.7 M-4.6 M of LG17, which is consistent with its location along the genetic map of pear (Yamamoto et al. 2007). Altogether six candidate $S F B$ genes within the $S$-locus are predicted and show high frequencies of amino acid polymorphisms, ranging from $61.7 \%$ to $76.1 \%$. As the scaffold containing $S$-RNase genes in pear is unanchored, an accurate estimation of the physical distance between $S$-RNase genes and the six candidate $S F B$ genes cannot be made (Supplemental Table 11). Comparisons of the $S$-locus region $(1000 \mathrm{~kb})$ for pear, apple, strawberry, and potato show that there are relatively moderate levels of synteny in this region (Fig. 5), and few common genes, except for S-RNase and SFB genes, are present in different plant species. Gene trafficking and rearrangements are active in this region. These findings indicate that the evolution of the $S$-locus region must have occurred following divergence of Rosaceae.

Unlike apple and strawberry, the six candidate $S F B$ genes are present as tandem repeats in pear. Thus, we propose that this unique characteristic of pear may be the result of gene duplication, thereby suggesting that a different mechanism for pollen self-incompatibility may be involved. Another interesting finding is the detection of highly repetitive sequences in $S$-locus regions of pear, apple, and potato, but not in strawberry, which exhibits selfcompatibility (Fig. 5). Suppression of recombination at the $S$-locus region may be related to presence of many repetitive sequences in pear. The function of repetitive sequences in GSI requires further studies. Moreover, different repetitive sequences may play a role in the evolution of the $S$-locus, as reported in Brassica species (Fujimoto et al. 2006).

\section{Biological processes underlying fruit quality}

\section{Stone cells}

Stone or grit cells, present in flesh of fruit, are important features of fruit quality in pear, but they are rare in other fruits. Lignin is the primary component of stone cells in pear fruit (Tao et al. 2009), and its synthesis has direct influence on formation and content of stone cells, ultimately influencing quality of pear fruit. By annotating the lignin biosynthesis pathway, it is revealed that lignin metabolism related genes in pear have similar levels of abundance to those found in apple and poplar, where lignin is involved in wood formation (Supplemental Table 12). Following phylogenetic analysis, 66 lignin synthesis-related gene families in pear show expansion, with pear exhibiting a greater demand for lignin synthesis. Predicted functions of transcription factors (TFs) involved in the lignin pathway have identified that gene numbers of NAC and LIM families (Supplemental Table 8), reported to be related to lignin synthesis (Kawaoka et al. 2000; Zhong et al. 2006), were more than those found in strawberry, grape, and papaya. These two forms of TFs may be involved in lignin formation in pear fruit.

To further pursue genomic analysis of lignin in pear fruit, RNA-seq data from three stages of fruit development (S422, early development; S627, middle development; and S830, near ripening) have been analyzed (Fig. 6). Genes involved in lignin synthesis are highly expressed in the first two stages, almost 10 -fold higher than those detected at or near ripening. Expression levels of genes encoding hydroxycinnamoyl transferases (HCT) are high at early stages of fruit development. HCT genes are known to promote lignin synthesis (Hoffmann et al. 2004), as companions to highly expressed genes encoding $p$-coumaroyl-shikimate/quinate $3^{\prime}$-hydroxylases $\left(\mathrm{C}^{\prime} \mathrm{H}\right)$ and caffeoyl-CoA O-methyltransferase (CCOMT), leading to high levels of conversion of $p$-coumaroylCoA (PCC) into caffeoyl-CoA (CFC) and feruloyl-CoA (FC) and resulting in accumulation of both G-lignin and S-lignin. These findings support the hypothesis that there are higher levels of G-lignin and S-lignin, but not of P-lignin, in pear stone cells. Meanwhile, none of the caffeic acid 3-O-methyltransferase (COMT) genes are expressed at all three stages of fruit development, suggesting that the rate limiting step for synthesis of lignin in pear fruit is that of conversion of CFC into FC (Fig. 6).

\section{Sugar}

The composition and content of sugar has an important influence on fruit quality and flavor. Instead of sucrose, which is common in nonrosacaeous species, sorbitol is a major photosynthetic product

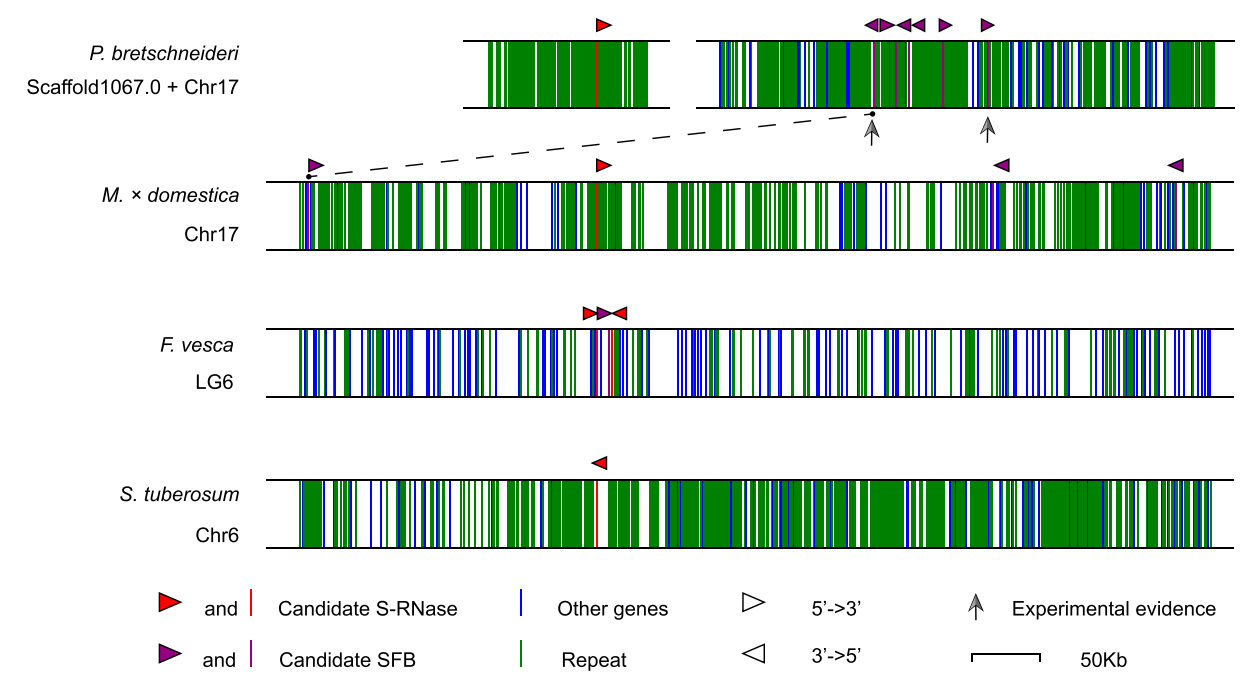

Figure 5. Genes and repeat sequences surrounding candidate S-RNase genes in pear, apple, strawberry, and potato. The dashed line with dot endpoints connects candidate SFB genes of pear and apple that share the highest sequence identity (which are shown in Supplemental Table 10). (Gray arrow) Genes supported by experimental evidence. The vertex of the triangles is the $3^{\prime}$ orientation of the particular gene: (red) candidate S-RNase gene; (purple) candidate SFB gene; (blue) other genes in the neighboring regions; (green) repeat sequence.

\section{Genome Research}



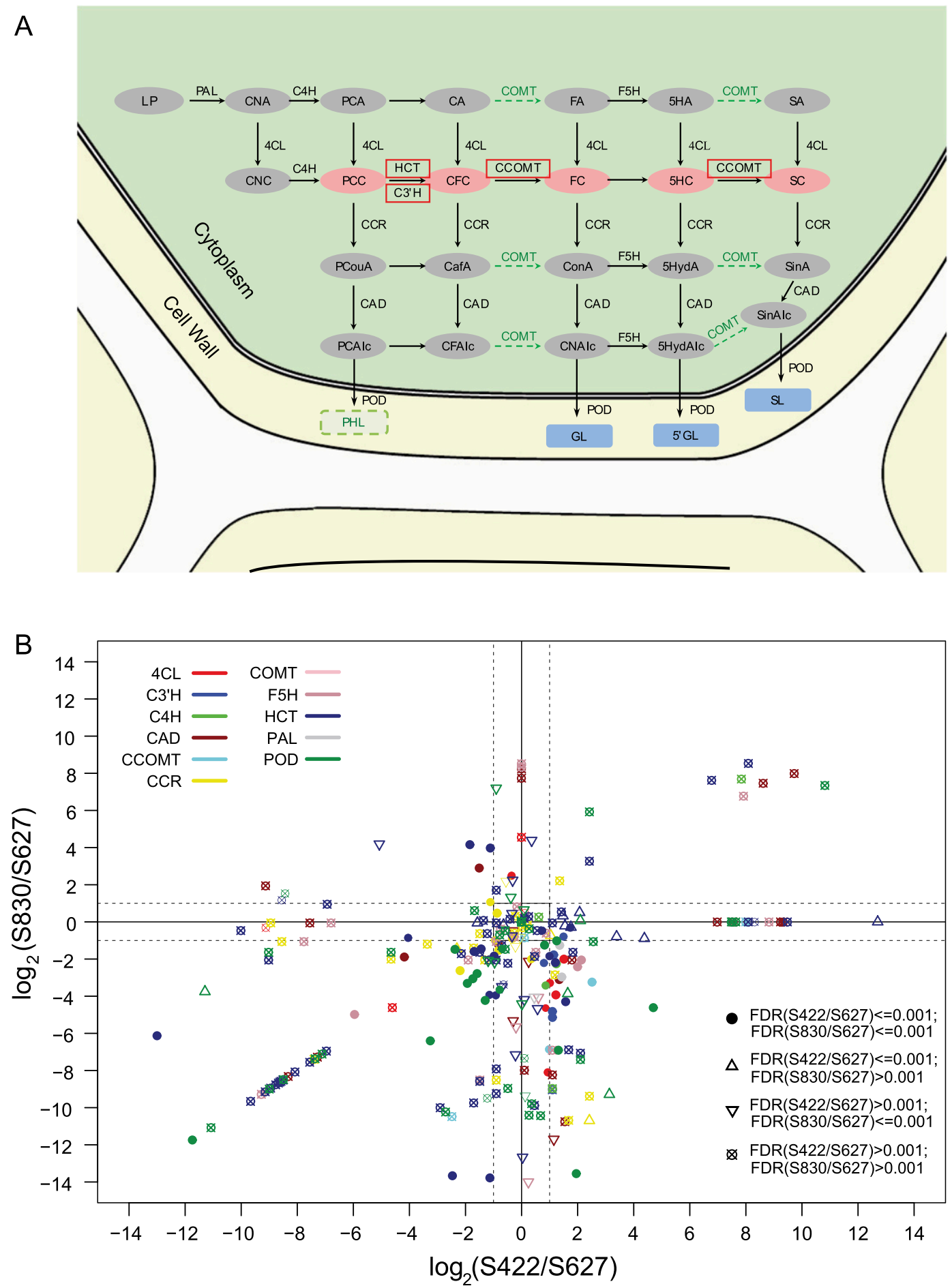

Figure 6. (A) The phenylpropanoid biosynthesis pathway in pear that influences the conformation of stone cells in fruit. (Red box) Genes that had detectable expression; (light red shaded ovals) important intermediate compounds in lignin pathway; (green text and arrows) pathways with minor expression; (blue boxes) three important end-product compounds in pear fruit; and green-boxed compound cannot be detected. (B) Transcript ratio distribution of all enzymes related to lignin. Three stages of fruit development (\$422, early development; S627, middle development; and S830, near ripening) were assessed. The ratio of S422 and S627 is shown along the $x$-axis, and the ratio of S830 and S627 is shown along the $y$-axis. For points, different colors correspond to different enzymes, while different shapes correspond to different conditions of false-discovery rate (FDR) values. Abbreviations of genes involved in phenylpropanoid biosynthesis pathway are as follows: LP, L-phenylalanine; CAN, cinnamic acid; PCA, P-coumaric acid; CA, caffeic acid; FA, ferulic acid; 5HA, 5-hydroxyferulate acid; SA, sinapic acid; CNC, cinnamoyl-CoA; PCC, p-coumaroyl-CoA; CFC, caffeoyl-CoA; FC, feruloylCoA; 5HC, 5-hydroxyferuloyl-CoA; SC, sinapoyl-CoA; PCouA, p-coumar aldehyde; CafA, caffeyl aldehyde; ConA, conifer aldehyde; 5HydA, 5-hydroxyconifer aldehyde; SinA, sinapoyl aldehyde; PCAlc, p-coumaryl alcohol; CFAlc, caffeyl alcohol; CNAlc, coniferyl alcohol; 5HydAlc, 5-hydroxyconiferyl alcohol; SinAlc, sinapyl alcohol; PHL, p-hydroxyphenyl lignin; GL, guaiacyl lignin; 5GL, 5-hydroxy-guaiacyl lignin; SL, syringyl lignin; PAL, phenylalanine ammonialyase; $\mathrm{C} 4 \mathrm{H}$, trans-cinnamate 4-monooxygenase; $4 \mathrm{CL}$, 4-coumarate-CoA ligase; $\mathrm{HCT}$, shikimate O-hydroxycinnamoyltransferase; $\mathrm{C}^{\prime} \mathrm{H}^{\prime}$, coumaroylquinate 3'-monooxygenase; COMT, caffeic acid 3-O-methyltransferase; CCOMT, caffeoyl-CoA O-methyltransferase; F5H, ferulate-5-hydroxylase; CCR, cinnamoylCoA reductase; $C A D$, cinnamyl-alcohol dehydrogenase; and POD, peroxidase. 
and phloem-translocated component in rosaceous fruit crops. A comparison of sorbitol metabolism-related genes in different species has revealed that the three gene families of sorbitol transport (SOT), sorbitol dehydrogenase (SDH), and sorbitol-6-phosphate dehydrogenase (S6PDH) in pear are higher than those in nonrosaceous species but are similar to those found in apple and strawberry (Supplemental Table 13; Supplemental Fig. 21). This indicates that duplication of the whole sorbitol metabolism pathway may have occurred to promote species fitness (van Hoek and Hogeweg 2009).

Gene families of $S 6 P D H, S D H$, and SOT have been expanded in both the pear and apple genomes, and all three gene families belong to the Maloideae-specific clade (Supplemental Table 13). Despite the close relationship between pear and apple, notable differences can still be found in the number of S6PDH genes, four members in pear compared with 11 members in apple (Fig. 7). In addition, the four S6PDH genes in pear are clustered into two clusters on chromosomes 5 and 2; however, in apple, there is only a single cluster located on chromosome 10, with others scattered on different chromosomes and scaffolds. These findings demonstrate that $S 6 P D H$ gene-expansion in apple or $S 6 P D H$ gene-contraction in pear must have occurred following their divergence from a common ancestor. Moreover, transcriptome data indicate that all four $S 6 P D H$ genes are expressed in fruit, thus indicating that sorbitol could also be resynthesized from monosaccharides, especially during later stages of fruit development. A total of 15 $S D H$ genes in pear are transcribed and clustered onto two homologous chromosomes, 1 and 7, along the same orientation. These are cross-paired on the phylogenetic tree (Supplemental Fig. 22), indicating that $S D H$ genes must have expanded mainly through WGD. Whereas, 15 SDH genes in apple are more scattered and oriented in different directions (Supplemental Fig. 23), suggesting that potential transposition events must have occurred. In addition, presence of pear-specific and apple-specific SOT genes in the phylogenetic tree (Supplemental Fig. 24) suggests that SOT genes have continued to expand following their divergence from the common Rosaceae ancestor.

\section{Volatiles}

Aroma is another important trait of pear fruit quality. Volatile compounds are mainly derived from the metabolism of fatty acids, amino acids, and carbohydrates (Schwab et al. 2008). When we compared all genes involved in three likely pathways of different plant genomes, we have found that lipoxygenase (LOX) and alcohol dehydrogenase $(\mathrm{ADH})$, both involved in the alpha-linolenic acid metabolism pathway, have higher numbers of genes in both pear and apple (Supplemental Table 14). Further RNA-seq data (Supplemental Fig. 25) have provided evidence that a third of $L O X$ homologous genes are highly expressed during fruit development,

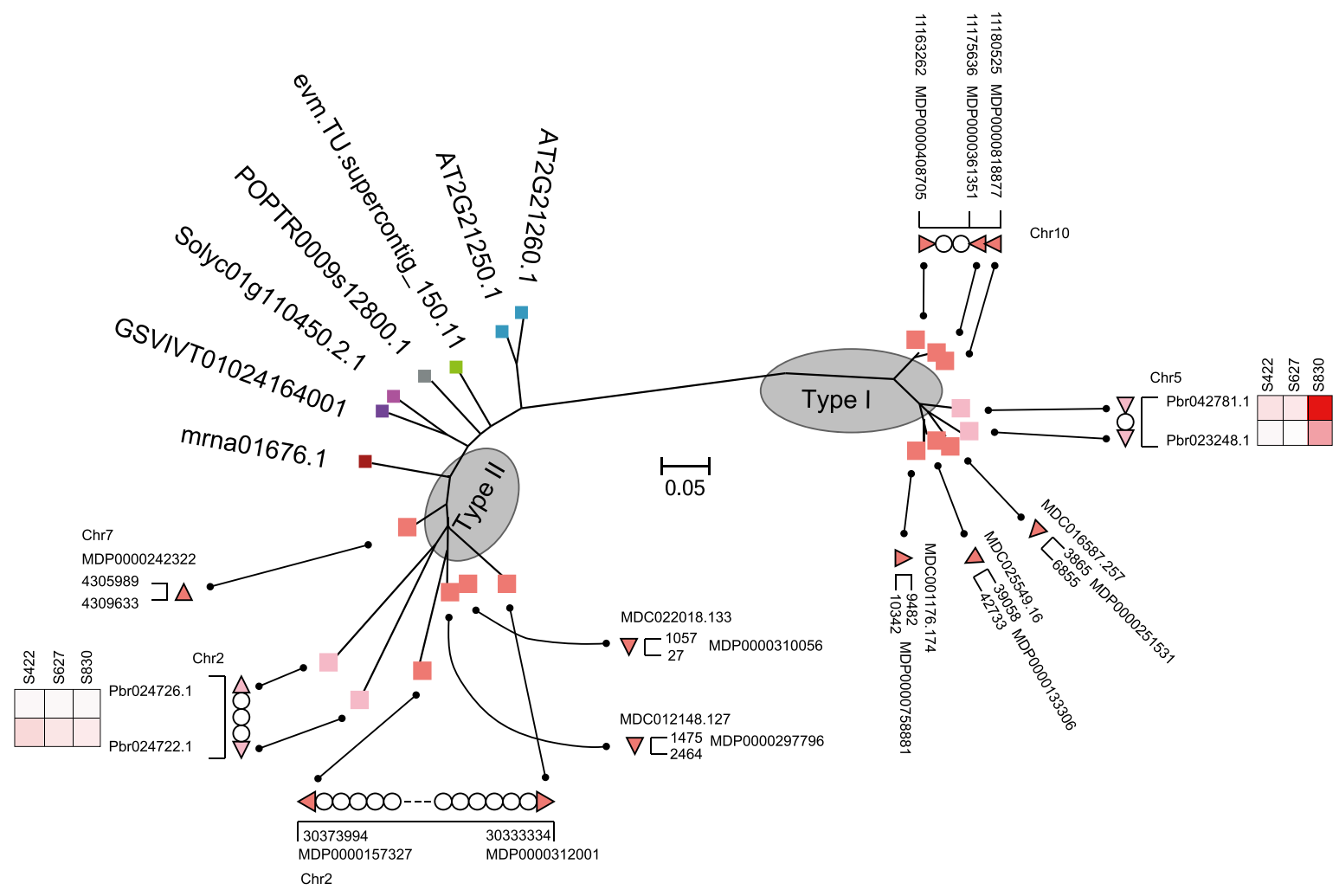

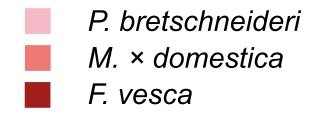

\begin{abstract}
$P$. bretschneideri
F. vesca
\end{abstract}

\begin{abstract}
A. thaliana
S. Iycopersicum

P. trichocarpa

V. vinifera

C. papaya
\end{abstract}

Figure 7. Phylogenetic relationships, distribution patterns, and transcriptional expression of S6PDH genes. The phylogenetic tree was constructed using the maximum likelihood method with Mega 5.0 software (Tamura et al. 2011). Heatmaps of expression patterns were drawn using Cluster 3.0 (de Hoon et al. 2004) along with expression levels (fragments per kilobase per million mapped reads [FPKM]) of each of the S6PDH genes. Different colors have been used for different species. S422, S627, and S830 are three different stages of development. Dotted lines between circles correspond to deleted non-S6PDH genes.

\section{Genome Research} www.genome.org 
reaching peak levels at the intermediate stage. Meanwhile, expression levels of $A D H$ increased along with alcohol formation during fruit development. Therefore the metabolism of alphalinolenic acid is likely to be important for aroma formation in pear.

The release of volatiles is another important aspect for perception of smell and flavor. The numbers of beta glucosidase homologous genes, which catalyze the release of aroma volatiles from glucose indicans, are high in both pear (101) and apple (158) (Supplemental Table 14). As not all 101 beta glucosidase genes have clustered with known beta glucosidase, there may be novel functional genes affecting aroma that are yet to be determined. Moreover, RNA-seq data (Supplemental Fig. 25) have revealed that only $20 \%$ of beta glucosidase homologous genes are expressed in pear fruit, and their expression levels have declined during fruit development. This has indicated that low aroma, perceived by sensory evaluation in pear, may be attributed to presence of more volatiles in bound status that are not released.

\section{Conclusions}

The sequenced pear genome will expedite basic research and crop improvement of this fruit crop. Advances in next-generation sequencing technologies have allowed genome sequencing to become accessible for crop plants; however, most perennial plant genomes are heterozygous, and assembling a heterozygous genome using WGS sequences is challenging and often results in inaccurate genome assembly. To overcome this limitation, a BACby-BAC approach is used in combination with the high-throughput sequencing technology to limit cost while ensuring the quality of the assembled genome. The BAC-by-BAC approach is labor-intensive, and for this project, a total of 76,608 Illumina sequencing libraries have been constructed, two libraries for each of the 38,304 BACs. The high quality of this genome is demonstrated by accurate annotation of genes and correction of 16,041 misannotated genes and redundant scaffolds in the apple draft genome using the WGS strategy. As for the nine ancestral chromosomes reported in apple, we infer that these nine chromosomes not only are the origin of the Pyreae tribe but also serve as the ancestors of the whole Rosaceae family.

The sequence of the pear genome provides an invaluable new resource for biological research of Pyrus. In this study, the pear genome and related transcriptome analysis have provided insights into mechanisms underlying important biological processes, including stone cell formation, sugar accumulation, and aroma formation and release. Availability of nearly all pear gene sequences should benefit researchers working on fruit quality, developmental controls, and disease resistance by enabling genome-wide functional studies and accelerating identification of gene-trait associations. In addition, further genome-wide comparative studies will provide insight and advance our knowledge on the genome evolution of Rosaceae. The high collinearity between pear and apple, combined with strawberry, provides more opportunity to reveal significant microsynteny, and the availability of the genome sequence will enable continued comparative genomics studies among species that will shed new light on gene family evolution.

\section{Methods}

\section{Genome sequencing}

A BAC-to-BAC strategy combined with WGS sequencing was employed in assembly of the genome sequence of pear. And we used Illumina Hiseq 2000 to sequencing the genome.
For BAC libraries construction, HindIII and BamHI were used to generate partially digested insert DNA, and these were ligated into appropriate sites of the vector pSMART (Lucigen). Ligations were transformed into phage-resistant Escherichia coli EPI-300 host cells. On average, insert sizes in these BAC libraries ranged between $80 \mathrm{~kb}$ and $180 \mathrm{~kb}$. For BAC clone DNA isolation, following culturing single colony in LB medium with antibiotics and growing $16-20 \mathrm{~h}$ at $37^{\circ} \mathrm{C}$, DNA was extracted and digested with NotI, Then, pulsed-field gel electrophoresis was used to separate transformed DNA from E. coli. The quality and quantity of BAC DNA were checked using an UV-VIS spectrometer along with gel electrophoresis runs of random samples. Usually, at least $0.75 \mu \mathrm{g} \mathrm{BAC}$ DNA was necessary for a single library preparation.

For BAC sequencing library construction, an Agilent Bravo Automated Liquid Handling Platform (Agilent) and an Agilent BenchCel Microplate Handler (Agilent) were used. Initially, the Adaptive Focused Acoustics (AFA) DNA fragmentation system (Covaris) was used to fractionate DNA samples based on insert sizes. For the automated batch processing capability, 96-microTUBE plates (Covaris) were used as sample vessels. Then, T4 DNA polymerase (Illumina) and E. coli DNA polymerase I Klenow fragment (Illumina) were used to convert overhangs resulting from fragmentation into blunt ends. To ligate index adapters (Supplemental Table 16), having single ' $\mathrm{T}$ ' base overhangs at 3 ' ends of DNA fragments, the polymerase activity of Klenow fragment was used to add ' $A$ ' bases to 3' ends of blunt DNA fragments. Following ligation, DNA samples with different index adapters were pooled together, according to the sample's position on the plate. Then, unligated index adapters were removed along an electrophoresis gel, and DNA segments of particular sizes were selected. Subsequently, index primers (Supplemental Table 16) were ligated to DNA segments, and PCR was used to selectively enrich those DNA fragments having index adapters and index primers on both ends and also to amplify the amount of DNA in the library. Then, gel electrophoresis was used to remove unligated index primers and to select DNA segments based on size. Finally, quality-control tests were conducted using Agilent 2100 Bioanalyzer (Agilent) and StepOnePlus Real-Time PCR System (ABI). Prior to sequencing, 23 96-well plates were pooled into a single lane (i.e., 2208 samples/ lane), resulting in an average throughput of $\sim 100.8 \mathrm{M}$ reads/lane (assuming a read length of $100 \mathrm{bp}$, adding up to $\sim 10 \mathrm{G}$ reads/lane). After sequencing, contamination from $E$. coli reads $(\sim 6 \%)$ were filtered out in raw data of each BAC prior to assembly.

For WGS sequencing library construction, a Illumina genomic DNA library construction protocol was used, and a total of 10 paired-end or mate-pair libraries, spanning sizes of $180 \mathrm{bp}$ to $40 \mathrm{~kb}$, were constructed (Supplemental Table 2). Most reads generated from mate-pair libraries (insert size $\geq 2000 \mathrm{bp}$ ) were in the order of $49 \mathrm{bp}$, whereas the corresponding length of paired-end libraries (ranging from 180 to $800 \mathrm{bp}$ ) was $100 \mathrm{bp}$.

For transcriptome sequencing, fruit samples at $15 \mathrm{~d}, 80 \mathrm{~d}$, and $145 \mathrm{~d}$ after flowering (DAF) were used. RNA sequencing libraries were constructed using an Illumina standard mRNA-Seq Prep Kit (TruSeq RNA and DNA Sample Preparation Kits version 2).

\section{Genome assembly and SNP calling}

The pear genome was assembled using short-read assembly software SOAPdenovo (http://soap.genomics.org.cn/) and sequence alignment software BLAT (Kent 2002). Scaffolds were constructed using SSPACE (Boetzer et al. 2011) software. First, each BAC was assembled with $\mathrm{K}=27$ by SOAPdenovo using pair-end reads $(250$ $\mathrm{bp}$ and $500 \mathrm{bp}$ ), and then WGS mate-pair reads were used to construct scaffolds by SSPACE. Later, assembled BAC sequences were mixed, and a seed sequence, $\sim 3 \mathrm{~kb}$ at ends of each scaffold, 
was selected to perform BLAT (Kent 2002) alignment for all scaffold sequences. Subsequently, similar sequences were combined and filtered for redundant bases using alignment results. If ends of scaffolds were aligned at high identity (90\%), they were merged into a single scaffold. Whereas if a short scaffold aligned at high identity to the interior of another scaffold, then the shorter scaffold was deleted. If sequences had mutual complementation, these were combined into a single scaffold. After several iterations of these steps, the whole sequence length tended to stabilize. Finally, scaffolds were further linked into super-scaffolds by mate-pair WGS reads $(2 \mathrm{~kb}-40 \mathrm{~kb})$ using SSPACE software (Boetzer et al. 2011), and gaps were filled with short read data.

The quality of the assembly was assessed by alignment to Sanger-derived phase 5 BAC sequences. By use of NUCmer software (http://mummer.sf.net/) to identify the scaffold related to the BAC, BACs were aligned to scaffolds using BLAST (Altschul et al. 1990). The SOAPaligner (http://soap.genomics.org.cn/soapaligner. $\mathrm{html}$ ) was used to map reads to BACs, and statistics were performed for each BAC.

WGS reads (insert size $<2 \mathrm{~K}$ ) were aligned to the genome using Bwa (Li and Durbin 2009), and SOAPsnp (http://soap.genomics. org.cn/soapsnp.html) was used to detect SNPs. Further filtering conditions were set as quality scores of the consensus genotype of more than 20, sequencing depth of the site of more than four and less than 120 , and with an average copy number of a nearby region of less than two.

\section{Development of RAD markers and anchoring of scaffolds}

Individual genetic maps, derived from an F1 population of a cross between 'Bayuehong' and 'Dangshansuli' and consisting of 102 individuals, were used to develop an integrated map for anchoring scaffolds.

Genomic DNA was isolated from fresh leaves using the plant genomics DNA Kit (TIANGEN), according to the manufacturer's recommendations. The restriction site-associated DNA (RAD) protocol (Chutimanitsakun et al. 2011) was used, except for the use of EcoRI (recognition site $5^{\prime} \mathrm{G}^{\wedge} \mathrm{AATTC} 3^{\prime}$ ). A total of $24 \mathrm{~F} 1 \mathrm{in}$ dividuals were pooled into a sequencing library with nucleotide multiplex identifiers ( $4 \mathrm{bp}, 6 \mathrm{bp}$, and $8 \mathrm{bp} ; \sim 1 \mathrm{~Gb}$ ), and 50-bp reads ( $9.94 \mathrm{Mb}$ reads data for each progeny on average) were generated on the NGS Illumina platform HiSeq2000. The SNP calling algorithm was done using a Stacks package (Catchen et al. 2011) with default parameters. SNP markers were filtered by testing against expected segregation ratios (1:2:1 or 1:1) using a chi-square test, and then their sequence reads were aligned to scaffolds by BLAT (Kent 2002). Only those unique aligned SNPs with a cutoff value of $90 \%$ identity were kept. Finally, all qualified SNP markers were used to construct the pear consensus map using the $\mathrm{CP}$ population option and the Kosambi mapping function in JoinMap version 3.0 (Van Ooijen and Voorrips 2001).

\section{Repeat sequences}

The Repbase (Repbase16.02) (Jurka et al. 2005) was used to find repeats by using RepeatProteinMask (Smit et al. 2004) and RepeatMasker (Smit et al. 2004). RepeatModeler (Smit et al. 2004) was used to build de novo repeats. Then redundancies were filtered out, and Repeatasker (Smit et al. 2004) was used to identify positions of repeats. Through structural features, LTR_Finder software (Xu and Wang 2007) and TRF software (Benson 1999) were used to find LTRs and tandem repeats, respectively.

For structure-based search of intact TEs, LTR retrotransposons were detected by LTR_Finder (Xu and Wang 2007) and LTR_STRUC (McCarthy and McDonald 2003). Insertion time of intact LTRs was estimated by computing with Dismat after measuring distances between 5' and 3' solo-LTRs. MITEs were detected by MITE_Hunter (Han and Wessler 2010). LINEs were detected by MGEScan nonLTR (Rho and Tang 2009). DDE domain TEs were detected by checking flanking sequence alignments of DDE domains that were identified by scanning plant TE domains.

\section{Genome and ncRNA annotation}

Augustus (Stanke et al. 2006) and GlimmerHMM (Majoros et al. 2004) were used to perform de novo prediction based on the repeat-masked genome. Homologous proteins of other plant species (apple, strawberry, grape, and Arabidopsis) were mapped to the genome using TBLASTN (Altschul et al. 1990) with an E-cutoff value of $1 \times 10^{-5}$. Aligned sequences, as well as their corresponding query proteins, were then filtered and passed to GeneWise (Birney et al. 2004) to accurately search for spliced alignments. Then, GLEAN (Elsik et al. 2007) was used to integrate these two sources of evidence to produce a consensus gene set.

About 7.8 Gb transcriptome sequence, of mixed multiple tissues generated by Illumina RNA-seq, was used to predict transcripts with TopHat (http://tophat.cbcb.umd.edu) and Cufflinks (http://cufflinks.cbcb.umd.edu). Then predicted transcripts were used to complement the GLEAN gene set or were integrated as isoforms. Novel genes were added to generate the final gene set.

tRNAscan-SE (Lowe and Eddy 1997) was performed to search for reliable tRNA positions. Searches for snRNA and miRNA were done through a two-step method: first aligned with BLAST and then searched with INFERNAL against the Rfam database (Griffiths-Jones, et al. 2005). rRNAs were detected by aligning with BLASTN against known plant rRNA sequences.

\section{Gene families and phylogenetic analyses}

Proteins of pear, apple, strawberry, grape, papaya, poplar, rice, and Arabidopsis were selected to perform all-against-all comparison using BLASTP (Altschul et al. 1990). The results were fed into the stand-alone OrthoMCL (Li et al. 2003) program using a default MCL inflation parameter of 1.5 . Single-copy families were selected to perform alignment by MUSCLE (Edgar 2004). Fourfold degenerate sites (4d) were picked by PhyML (Guindon et al. 2010), based on the maximum likelihood method (Guindon and Gascuel 2003), to reconstruct the phylogenetic tree using rice as an outgroup. The divergence time was estimated by MultiDivtime (Edgar 2004) using the divergence time of papaya and Arabidopsis, identified by fossil records (Crepet et al. 2004). Subsequently, CAFÉ (De Bie et al. 2006) was used to identify gene family expansion and contraction.

\section{Collinearity and WGD}

MCscan (Tang et al. 2008) was used to identify collinearity blocks using paralog gene pairs, which were then identified by BLASTP (Altschul et al. 1990). Through MUSCLE alignment of gene pairs in collinearity blocks (Edgar 2004), 4dTv (transversion of fourfold degenerate site) values of each block were calculated using the sum of transversion of fourfold degenerate sites divided by the sum of fourfold degenerate sites (Huang et al. 2009). Ks values were calculated using MUSCLE (Edgar 2004) alignment and PAML (Yang 2007) for gene families of paralogous gene pairs. All these values were used for WGD analysis.

\section{Disease resistance genes}

Identification of pear resistance-related genes was based on the most conserved motif structures of plant resistance proteins, in- 
cluding CC, KIN, TIR, NBS, and LRR finger domains. Conserved motifs were derived from domain profiles retrieved from the PFAM, PANTHER, PRINTS, PROSITE, SMART, and SUPERFAMILY databases and from PAIRCOIL2 (McDonnell et al. 2006) to specifically detect CC domains. 'Other' types of $R$ genes (without most conserved motifs, nevertheless potential $R$ genes) were determined by BLAST (Altschul et al. 1990) based on a threshold of $60 \%$ similarity using the 'Other'-type reference of PRGDB (Sanseverino et al. 2010) as a reference sequence. Assigning candidate genes to different $R$ classes was based on the aforementioned protein domain composition. $R$ genes were grouped into clusters when they were not interrupted by more than eight other ORFs encoding non- $R$ proteins.

\section{Data access}

The whole-genome sequences of the pear (P. bretschneideri Rehd.) project have been deposited at GenBank (http://www.ncbi.nlm. nih.gov/genbank) under accession no. AJSU00000000. The version described in this article is the first version AJSU01000000. The data are also available at our professional website (http:// peargenome.njau.edu.cn).

\section{Acknowledgments}

This work was supported by the National Natural Science Foundation of China (30900974, 31071759, 31000888, 31171928, and 31171936), the Fundamental Research Funds for the Central Universities (KYZ200911, KYZ201146), and the earmarked fund for the China Agriculture Research System (CARS-29).

Author contributions: S-L. Zhang, J. Wu, and J. Wang managed the project. S-L. Zhang, J. Wu, Z-B. Shi, S. Zhang, Z-W. Wang, R. Ming, M. A. Khan, S. S. Korban, X. Xu, and T. Nishio designed the analyses. J. Wu, K-J. Qi, H. Yin, C. Gu, Y-N. Yang, Y-T. Wang, W-J. Huang, M-S. Dai, Y-Z. Wang, and S-J. Zhang collected samples and prepared DNA and RNA. Z-W. Wang, J. Wu, S-L. Zhu, S-T.Tao, X. Zhao, L. Cong, J. L. Bennetzen, N. Chen, R. E. Paull, H. Wang, X-W. Wang, C. Deng, Y-H. Sha, D-H. Shi, H. Yin, J-Y. Wu, G-H. Qin, H-P. Zhang, S-F. Sha, C-L. Wang, Y-Z. Wang, C-Y. Gou, W-L. Zhou, D-L. Zhan, J. Wang, M-S. Chen, G-B. Wang, L. Xie, J-Y. Wang, and S-L. Zhang contributed to sequencing, sequence assembly, genome annotation, genome structure, evolution and pathway analyses. J. Wu, H. Chen, Y. Song, Y. Tao, L. Zeng, L-T. Li, D. Zheng, M. A. Khan, M-Y. Zhang, J-M. Sun, L-L. Xu, Y. Li, X. Liu, Q-S. Li, J-H. Shen, and S-L. Zhang contributed to genetic mapping and chromosome anchoring. J. Wu, Z-W. Wang, S-L. Zhu, X-S. Huang, Valpuri Sovero, R. E. Paull, S. S. Korban, M. A. Khan, R. Ming, and S-L. Zhang wrote the paper.

\section{References}

Al-Dous EK, George B, Al-Mahmoud ME, Al-Jaber MY, Wang H, Salameh YM, Al-Azwani EK, Chaluvadi S, Pontaroli AC, DeBarry J, et al. 2011. De novo genome sequencing and comparative genomics of date palm (Phoenix dactylifera). Nat Biotechnol 29: 521-527.

Altschul SF, Gish W, Miller W, Myers EW, Lipman DJ. 1990. Basic local alignment search tool. J Mol Biol 215: 403-410.

The Arabidopsis Genome Initiative. 2000. Analysis of the genome sequence of the flowering plant Arabidopsis thaliana. Nature 408: 796-815.

Bennetzen JL, Schmutz J, Wang H, Percifield R, Hawkins J, Pontaroli AC, Estep M, Feng L, Vaughn JN, Grimwood J, et al. 2012. Reference genome sequence of the model plant Setaria. Nat Biotechnol 30: 555-561.

Benson G. 1999. Tandem repeats finder: A program to analyze DNA sequences. Nucleic Acids Res 27: 573-580.

Birney E. 2010. Assemblies: The good, the bad, the ugly. Nat Methods 8: 59-60.

Birney E, Clamp M, Durbin R. 2004. GeneWise and genomewise. Genome Res 14: $988-995$
Boetzer M, Henkel CV, Jansen HJ, Butler D, Pirovano W. 2011. Scaffolding pre-assembled contigs using SSPACE. Bioinformatics 27: 578-579.

Catchen JM, Amores A, Hohenlohe P, Cresko W, Postlethwait JH. 2011. Stacks: Building and genotyping loci de novo from short-read sequences. G3 1: 171-182.

Chutimanitsakun Y, Nipper RW, Cuesta-Marcos A, Cistué L, Corey A, Filichkina T, Johnson EA, Hayes PM. 2011. Construction and application for QTL analysis of a restriction site associated DNA (RAD) linkage map in barley. BMC Genomics 12: 4 .

Crepet WL, Nixon KC, Gandolfo MA. 2004. Fossil evidence and phylogeny: The age of major angiosperm clades based on mesofossil and macrofossil evidence from Cretaceous deposits. Am J Bot 91: 1666-1682.

De Bie T, Cristianini N, Demuth JP, Hahn MW. 2006. CAFE: A computational tool for the study of gene family evolution. Bioinformatics 22: 12691271

de Hoon MJ, Imoto S, Nolan J, Miyano S. 2004. Open source clustering software. Bioinformatics 20: 1453-1454.

de Nettancourt D. 1997. Incompatibility in angiosperms. Sex Plant Reprod 10: $185-199$

Edgar RC. 2004. MUSCLE: Multiple sequence alignment with high accuracy and high throughput. Nucleic Acids Res 32: 1792-1797.

Elsik CG, Mackey AJ, Reese JT, Milshina NV, Roos DS, Weinstock GM. 2007. Creating a honey bee consensus gene set. Genome Biol 8: R13. doi: 10.1186/gb-2007-8-1-r13.

Fawcett JA, Maere S, Van de Peer Y. 2009. Plants with double genomes might have had a better chance to survive the Cretaceous-Tertiary extinction event. Proc Natl Acad Sci 106: 5737-5742.

Fujimoto R, Okazaki K, Fukai E, Kusaba M, Nishio T. 2006. Comparison of the genome structure of the self-incompatibility (S) locus in interspecific pairs of $S$ haplotypes. Genetics 173: 1157-1167.

Griffiths-Jones S, Moxon S, Marshall M, Khanna A, Eddy SR, Bateman A. 2005. Rfam: Annotating non-coding RNAs in complete genomes. Nucleic Acids Res 33: D121-D124.

Guindon S, Gascuel O. 2003. A simple, fast, and accurate algorithm to estimate large phylogenies by maximum likelihood. Syst Biol 52: 696704.

Guindon S, Dufayard JF, Lefort V, Anisimova M, Hordijk W, Gascuel O. 2010. New algorithms and methods to estimate maximum-likelihood phylogenies: Assessing the performance of PhyML 3.0. Syst Biol 59: 307321.

Han Y, Wessler SR. 2010. MITE-Hunter: A program for discovering miniature inverted-repeat transposable elements from genomic sequences. Nucleic Acids Res 38: e199.

Hoffmann L, Besseau S, Geoffroy P, Ritzenthaler C, Meyer D, Lapierre C, Pollet B, Legrand M. 2004. Silencing of hydroxycinnamoyl-coenzyme A shikimate/quinate hydroxycinnamoyltransferase affects phenylpropanoid biosynthesis. Plant Cell 16: 1446-1465

Huang S, Li R, Zhang Z, Li L, Gu X, Fan W, Lucas WJ, Wang X, Xie B, Ni P. 2009. The genome of the cucumber, Cucumis sativus L. Nat Genet 41: $1275-1281$.

Jaillon O, Aury JM, Noel B, Policriti A, Clepet C, Casagrande A, Choisne N, Aubourg S, Vitulo N, Jubin C. 2007. The grapevine genome sequence suggests ancestral hexaploidization in major angiosperm phyla. Nature 449: 463-467.

Jurka J, Kapitonov VV, Pavlicek A, Klonowski P, Kohany O, Walichiewicz J. 2005. Repbase Update, a database of eukaryotic repetitive elements. Cytogenet Genome Res 110: 462-467.

Kawaoka A, Kaothien P, Yoshida K, Endo S, Yamada K, Ebinuma H. 2000. Functional analysis of tobacco LIM protein Ntlim1 involved in lignin biosynthesis. Plant J 22: 289-301.

Kent WJ. 2002. BLAT: The BLAST-like alignment tool. Genome Res 12: 656664.

Khurana P, Gaikwad K. 2005. The map-based sequence of the rice genome. Nature 436: 793-800.

Krzywinski M, Schein J, Birol I, Connors J, Gascoyne R, Horsman D, Jones SJ, Marra MA. 2009. Circos: An information aesthetic for comparative genomics. Genome Res 19: 1639-1645.

Li H, Durbin R. 2009. Fast and accurate short read alignment with BurrowsWheeler transform. Bioinformatics 25: 1754-1760.

Li L, Stoeckert CJ Jr, Roos DS. 2003. OrthoMCL: Identification of ortholog groups for eukaryotic genomes. Genome Res 13: 2178-2189.

Lombard PB, Westwood MN. 1987. Pear rootstocks. In Rootstocks for fruit crops (ed. RC Rom, RF Carlson), pp. 145-183. Wiley, New York.

Lowe TM, Eddy SR. 1997. tRNAscan-SE: A program for improved detection of transfer RNA genes in genomic sequence. Nucleic Acids Res 25: 955964

Majoros W, Pertea M, Salzberg S. 2004. TigrScan and GlimmerHMM: Two open source $a b$ initio eukaryotic gene-finders. Bioinformatics 20: 2878-2879.

McCarthy EM, McDonald JF. 2003. LTR_STRUC: A novel search and identification program for LTR retrotransposons. Bioinformatics 19: 362 367. 
McDonnell AV, Jiang T, Keating AE, Berger B. 2006. Paircoil2: Improved prediction of coiled coils from sequence. Bioinformatics 22: 356-358

Ming R, Hou S, Feng Y, Yu Q, Dionne-Laporte A, Saw JH, Senin P, Wang W, Ly BV, Lewis KLT. 2008. The draft genome of the transgenic tropical fruit tree papaya (Carica papaya Linnaeus). Nature 452: 991-996.

Rho M, Tang H. 2009. MGEScan-non-LTR: Computational identification and classification of autonomous non-LTR retrotransposons in eukaryotic genomes. Nucleic Acids Res 37: e143.

Rubtsov G. 1944. Geographical distribution of the genus Pyrus and trends and factors in its evolution. Am Nat 78: 358-366.

Sanseverino W, Roma G, De Simone M, Faino L, Melito S, Stupka E, Frusciante L, Ercolano MR. 2010. PRGdb: A bioinformatics platform for plant resistance gene analysis. Nucleic Acids Res 38: D814-D821.

Schwab W, Davidovich-Rikanati R, Lewinsohn E. 2008. Biosynthesis of plant-derived flavor compounds. Plant J 54: 712-732.

Shulaev V, Sargent DJ, Crowhurst RN, Mockler TC, Folkerts O, Delcher AL, Jaiswal P, Mockaitis K, Liston A, Mane SP. 2010. The genome of woodland strawberry (Fragaria vesca). Nat Genet 43: 109-116.

Smit AFA, Hubley R, Green P. 2004. RepeatMasker Open-3.0.

Stanke M, Keller O, Gunduz I, Hayes A, Waack S, Morgenstern B. 2006. AUGUSTUS: $a b$ initio prediction of alternative transcripts. Nucleic Acids Res 34: W435-W439.

Tamura K, Peterson D, Peterson N, Stecher G, Nei M, Kumar S. 2011. MEGA5: Molecular evolutionary genetics analysis using maximum likelihood, evolutionary distance, and maximum parsimony methods. Mol Biol Evol 28: 2731-2739.

Tang H, Bowers JE, Wang X, Ming R, Alam M, Paterson AH. 2008. Synteny and collinearity in plant genomes. Science 320: $486-488$.

Tao S, Khanizadeh S, Zhang H, Zhang S. 2009. Anatomy, ultrastructure and lignin distribution of stone cells in two Pyrus species. Plant Sci 176: $413-$ 419.

Tuskan GA, Difazio S, Jansson S, Bohlmann J, Grigoriev I, Hellsten U, Putnam N, Ralph S, Rombauts S, Salamov A. 2006. The genome of black cottonwood, Populus trichocarpa (Torr. \& Gray). Science 313: 1596-1604.

Ushijima K, Sassa H, Dandekar AM, Gradziel TM, Tao R, Hirano H. 2003. Structural and transcriptional analysis of the self-incompatibility locus of almond: Identification of a pollen-expressed F-box gene with haplotype-specific polymorphism. Plant Cell 15: 771-781. van Hoek MJA, Hogeweg P. 2009. Metabolic adaptation after whole genome duplication. Mol Biol Evol 26: 2441-2453.

Van Ooijen J, Voorrips R. 2001. JoinMap 3.0, Software for the calculation of genetic linkage maps, pp. 1-51. Plant Research International, Wageningen, The Netherlands.

Varshney RK, Chen W, Li Y, Bharti AK, Saxena RK, Schlueter JA. 2012. Draft genome sequence of pigeonpea (Cajanus cajan), an orphan legume crop of resource-poor farmers. Nat Biotechnol 30: 83-89.

Vavilov NI. 1951. The origin, variation, immunity and breeding of cultivated plants. Soil Sci 72: 482 .

Velasco R, Zharkikh A, Affourtit J, Dhingra A, Cestaro A, Kalyanaraman A, Fontana P, Bhatnagar SK, Troggio M, Pruss D, et al. 2010. The genome of the domesticated apple (Malus $\times$ domestica Borkh.). Nat Genet 42: 833839.

Wu J, Gu C, Zhang S, Zhang S, Wu H, Heng W. 2009. Identification of S-haplotype-specific S-RNase and SFB alleles in native Chinese apricot (Prunus armeniaca L.). J Hortic Sci Biotechnol 84: 645-652.

$\mathrm{Xu} \mathrm{Z}$, Wang H. 2007. LTR_FINDER: An efficient tool for the prediction of full-length LTR retrotransposons. Nucleic Acids Res 35: W265-W268.

Yamamoto T, Kimura T, Terakami S, Nishitani C, Sawamura Y, Saito T, Kotobuki K, Hayashi T. 2007. Integrated reference genetic linkage maps of pear based on SSR and AFLP markers. Breed Sci 57: 321-329.

Yang Z. 2007. PAML 4: Phylogenetic analysis by maximum likelihood. Mol Biol Evol 24: 1586-1591.

Yang L, Bennetzen JL. 2009. Distribution, diversity, evolution and survival of Helitrons in the maize genome. Proc Natl Acad Sci 106: 19922-19927.

Zeven AC, Zhukovsky PM. 1975. Dictionary of cultivated plants and their centres of diversity, pp. 62-63. Centre for Agricultural Publishing and Documentation, Wageningen, The Netherlands.

Zhang SL, Huang SX, Kitashiba H, Nishio T. 2007. Identification of S-haplotype-specific F-box gene in Japanese plum (Prunus salicina Lindl.). Sex Plant Reprod 20: 1-8.

Zhong R, Demura T, Ye ZH. 2006. SND1, a NAC domain transcription factor, is a key regulator of secondary wall synthesis in fibers of Arabidopsis. Plant Cell 18: 3158-3170.

Received June 15, 2012; accepted in revised form November 6, 2012. 


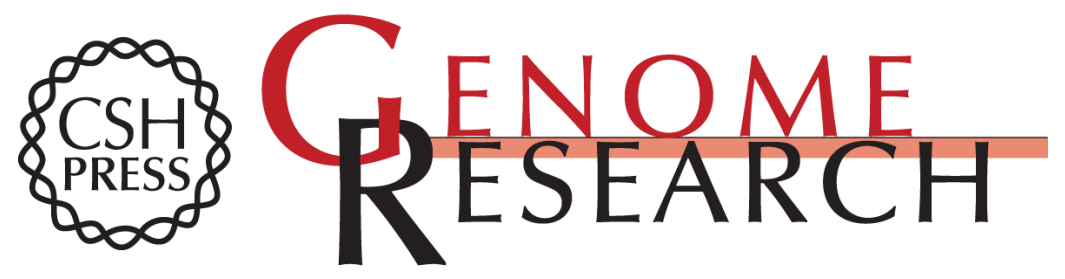

\section{The genome of the pear (Pyrus bretschneideri Rehd.)}

Jun Wu, Zhiwen Wang, Zebin Shi, et al.

Genome Res. 2013 23: 396-408 originally published online November 13, 2012

Access the most recent version at doi:10.1101/gr.144311.112

Supplemental http://genome.cshlp.org/content/suppl/2012/11/29/gr.144311.112.DC1
Material

References This article cites 58 articles, 13 of which can be accessed free at: http://genome.cshlp.org/content/23/2/396.full.html\#ref-list-1

Open Access Freely available online through the Genome Research Open Access option.

Creative This article is distributed exclusively by Cold Spring Harbor Laboratory Press for the Commons first six months after the full-issue publication date (see

License http://genome.cshlp.org/site/misc/terms.xhtml). After six months, it is available under a Creative Commons License (Attribution-NonCommercial 3.0 Unported License), as described at http://creativecommons.org/licenses/by-nc/3.0/.

Email Alerting Receive free email alerts when new articles cite this article - sign up in the box at the Service top right corner of the article or click here.

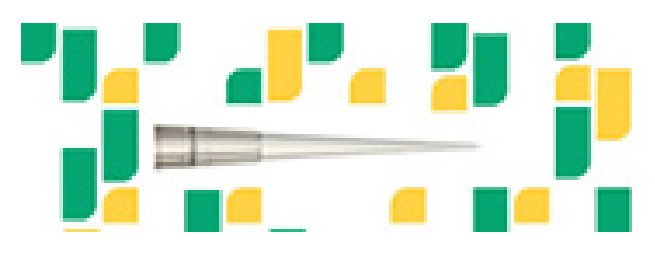

Focused on your science.

To subscribe to Genome Research go to:

https://genome.cshlp.org/subscriptions 